\section{Advantages and disadvantages of direct oral anticoagulants in older patients}

\author{
Antonio Cherubini, ${ }^{1}$ Barbara Carrieri, ${ }^{1,2}$ \\ Paolo Marinelli ${ }^{1,3}$ \\ ${ }^{1}$ Geriatric Emergency Care and Research \\ Center on Aging, IRCCS-INRCA, \\ Ancona; ${ }^{2}$ Department of Life and \\ Environmental Sciences, Polytechnic \\ University of Marche, Ancona; \\ ${ }^{3}$ Department of Experimental Medicine \\ And Public Health, University of \\ Camerino, Camerino (MC), Italy
}

\begin{abstract}
Atrial fibrillation (AF) and venous thromboembolism (VTE), which includes deep vein thrombosis and pulmonary embolism, are conditions that increase with age. Anticoagulant therapy is strongly recommended both in patients with AF for the prevention of cardioembolic stroke, and for treatment of VTE and prevention of recurrent VTE. Until recently, vitamin K antagonists (VKAs) were the only oral drugs for long-term anticoagulation. In the past decade, four direct oral anticoagulants (DOACs) were approved: a direct thrombin inhibitor (dabigatran) and three factor $\mathrm{Xa}$ inhibitors (apixaban, rivaroxaban, edoxaban). Despite increasing evidence demonstrating the efficacy and safety of DOACs in older patients, there are still gray areas where the use of VKAs might be valuable.
\end{abstract}

\section{Introduction}

Atrial fibrillation (AF) is a very common disease in the elderly, whose incidence and prevalence increase with age, affecting about $10 \%$ of subjects aged 80 years or older. AF is associated with severe complications, such as stroke and systemic thromboembolism. Oral anticoagulant therapy (OAT) is strongly recommended for the prevention of these negative events in patients with $\mathrm{AF}$ and intermediate or high risk, i.e. men with a score 1 and women 2 , according to the $\mathrm{CHA}_{2} \mathrm{DS}_{2}$-VASc score. Moreover, antiplatelet therapy is no longer recommended. ${ }^{1}$ Venous thromboembolism (VTE), which comprises deep vein thrombosis (DVT) and pulmonary embolism (PE), occurs in about $0.1 \%$ of the population each year. This figure increases exponentially with age, involving up to five times more often octogenarians. ${ }^{2}$ Anticoagulants are the first choice therapy for initial treatment of VTE as well as to prevent recurrent VTE.

For many decades vitamin $\mathrm{K}$ antagonists (VKAs) were the only oral drugs available for long-term anticoagulation. Several studies including RCTs, observational and real world studies in different clinical settings showed that warfarin reduces stroke incidence, mortality as well as the burden of post-stroke disability. Despite this, anticoagulant therapy is underused in older patients, due to several reasons, such as high risk of bleeding, comorbidities, disability, adherence issues, polypharmacotherapy, swallowing disorders, dementia, high risk of fall, difficulties in monitoring VKAs-based therapies, and short life expectancy. ${ }^{3}$

It is well known that VKAs have important disadvantages, including a narrow therapeutic range with necessity of regular monitoring of international normalized ratio (INR) and consequent dose adjustment, frequent food-drug interactions and drug-drug interactions potentially contributing to a higher incidence of adverse drug reactions. Recently, four direct oral anticoagulants (DOACs) have been approved for the prevention of thromboembolic risk in $\mathrm{AF}$ and treatment of VTE: a direct thrombin inhibitor (dabigatran) and three factor $\mathrm{Xa}$ inhibitors (apixaban, rivaroxaban, edoxaban). Overall use of anticoagulants has increased over the years, mainly due to the availability of DOACs. ${ }^{4}$

\section{Direct oral anticoagulants effectiveness and safety in atrial fibrillation and venous thromboembolism}

The efficacy and safety of DOACs compared to VKAs in older patients with AF and VTE have been compared in several systematic reviews and meta-analyses. ${ }^{5}$

Sardar and colleagues performed a meta-analysis of ten randomized controlled trials (RCTs) including 25,031 older subjects aged at least 75 years. DOACs (rivaroxaban, apixaban, and dabigatran) had higher efficacy in stroke or systemic embolism prevention than conventional therapy (i.e. VKAs, low molecular weight heparin, aspirin or placebo) in older adults with AF, while the risk of major or clinically relevant bleeding was similar. Moreover, DOACs treatment reduced the risk of venous thromboembolism (VTE) or death. ${ }^{6}$

Another systematic review, which considered only older adults over the age of 75
Correspondence: Antonio Cherubini, Geriatric Emergency Care and Research Center on Aging, IRCCS-INRCA, Ancona, Italy.

Tel.: +39.0718003537

Fax: +39.0718003337

E-mail: a.cherubini@inrca.it

Key words: Vitamin K antagonist; direct oral anticoagulant; prevention; thrombosis; bleeding risk.

Conference presentation: paper presented at $31^{\circ}$ Congresso Nazionale SIGOT - Genova, June 8-9, 2017.

Contributions: the authors contributed equally.

Conflict of interests: the authors declare no conflict of interests.

Received for publication: 7 December 2017. Accepted for publication: 4 February 2018.

This work is licensed under a Creative Commons Attribution-NonCommercial 4.0 International License (CC BY-NC 4.0).

(C) Copyright A. Cherubini et al., 2018

Licensee PAGEPress, Italy

Geriatric Care 2018; 4:7227

doi:10.4081/gc.2018.7227

years, found that DOACs were similar or better than VKAs in reducing thrombotic events, although noticeable differences were present in bleeding risk. Dabigatran at the highest dose, i.e. $150 \mathrm{mg}$, was associated with a higher probability of major bleeding compared to VKAs, although the difference did not reach the statistical significance. Moreover, lower bleeding risk was found in patients treated with apixaban and edoxaban, and rivaroxaban showed similar risk to VKAs.?

Sadlon et al. in a similar meta-analysis, which included 30,655 patients aged 75 or older, confirmed that DOACs have similar or higher efficacy compared to VKAs with a reduced bleeding risk. ${ }^{8}$

In a recent systematic review and metaanalysis including both randomized controlled trials and observational studies, DOACs were better than warfarin for stroke and VTE prevention while being associated with a reduced risk of major bleedings as well as mortality. ${ }^{9}$

RCTs apply very restrictive inclusion and exclusion criteria that may exclude many patients who could be encountered in real-life clinical practice (e.g. frail, with severe comorbidities and/or polypharmacy, with reduced life-expectancy). Real world studies analyzed routine clinical use of DOACs in a variety of national administrative, health insurance or proprietary data- 
base and registries to provide clinicians more accurate information that can be translate to routine practice. ${ }^{9}$

In the cited review by Bai a subgroup analysis showed that DOACs were superior to warfarin in the RCTs and real world studies for the reduction of stroke and VTE, while the risk of major bleeding was reduced in RTCs but not in the real world analyses. ${ }^{9}$ In particular, dabigatran and rivaroxaban had higher efficacy compared with warfarin in real world studies but also higher probability of bleeding, while the opposite was shown for apixaban and edoxaban.

Real life studies suggest also a good adherence in people treated with DOACs. ${ }^{10,11}$

\section{Advantages of direct oral anticoagulants in the elderly: a brief review}

Besides having a similar or superior efficacy and, in many instances, a better safety profile, DOACs present several other advantages as compared to VKAs: they have a wide therapeutic window and low interaction with foods, and therefore dietary restrictions are not required. ${ }^{12}$ The interactions between DOACs and other drugs are less common than with VKAs, but still there are some that are important to take into account. The risk of bleeding increases with the coadministration of DOACs and antiplatelet agents, other anticoagulants, non-steroidal anti-inflammatory drugs, some antidepressants (e.g. SSRI, SNRI), high dose vitamin E supplements. Moreover, all DOACs are a substrate of a P-gp transporter so the concomitant use of P-pg inhibitors such as amiodarone and verapamil may increase their serum concentration, on the other hand P-pg inducers such as phenytoin, rifampicin may decrease serum concentration. Apixaban and rivaroxaban and are in part metabolized by CPY3A4, so co-administration of inhibitors or inducers of CPY3A4 (e.g. ketoconazole, amiodarone, verapamil, rifampicin, clarithromycin, ritonavir and diltiazem) is not recommended because increase or decrease serum concentration. ${ }^{13}$ On the other hand edoxaban undergoes minimal metabolism by CPY3A4 (less than $10 \%)$, so the interaction with inhibitors or inducers of CPY3A4 are not relevant. With DOACs routine laboratory monitoring of coagulation is not necessary except in emergency conditions. The pharmacokinetics and pharmacodynamics is predictable and therefore they can be used with a fixed dosage, but studies in oldest old or frail patients are still lacking. DOACs are characterized by a rapid onset and offset and short half-life, so switching patients from low molecular weight heparins (LMWH) and VKAs to DOACs is easier. ${ }^{12}$

\section{Disadvantages of direct oral anticoagulants: a brief review}

DOACs should be prescribed with more caution in some patients, such as who have kidney or liver disease. Almost $80 \%$ of dabigatran and $33 \%$ and $25 \%$ of rivaroxaban and apixaban respectively are cleared by the kidneys as an active drug. Considering the high prevalence of renal insufficiency in older patients and the frequency of acute decrease, it is important that renal function is evaluated before prescribing and monitored more frequently during therapy with DOACs to avoid risk of bleendig. ${ }^{12}$ Regulatory agencies suggest that dabigatran $150 \mathrm{mg}$ should not be administered in patient aged 80 years, a lower dose of rivaroxaban and edoxaban should be used in subjects with creatinine clearance $15-45 / 50 \mathrm{~mL} / \mathrm{min}$, or in patients aged 80 years with body weight $60 \mathrm{~kg}$ and creatinine $1.5 \mathrm{mg} / \mathrm{dl}$ for apixaban. ${ }^{14}$ Therefore, in the presence of severe kidney impairment, e.g. terminal insufficiency or dialysis VKAs might be still preferable. Apixaban and rivaroxaban should be administered carefully in patients who have liver insufficiency or impairment, a dose adjustments may be required. Furthermore, there are not antidotes available for DOACs, except idarucizumab, which is effective for dabigatran, and this is a problem in the case of overdose, major bleeding or urgent surgical intervention. ${ }^{12}$ The rapid offset and short half-life in some patients could be detrimental if adherence is not optimal because it could expose patients to greater embolic risk, which is amplified by the absence of simple and standardized laboratory tests to monitor the anti-coagulation. There are also some issues for patients with dysphagia, e.g. dabigatran pills cannot be broken or chewed. Moreover, the high cost is an important disadvantage of the DOACs. ${ }^{15}$ Last but not least, the efficacy and safety of anticoagulants, VKAs or DOACs in frail patients remain not established, because these patients have largely been excluded from both randomized trials and real-world studies. ${ }^{16}$

In conclusion, DOACs provide an effective and safe treatment for many older patients with increased thrombotic risk, representing a valuable and often better therapeutic option compared to VKAs.

\section{References}

1. Kirchhof P, Benussi S, Kotecha D, et al. 2016 ESC Guidelines for the management of atrial fibrillation developed in collaboration with EACTS. Eur Heart J 2016;37:2893-962.

2. White RH. The epidemiology of venous thromboembolism. Circulation 2003; 107:I4-8.

3. Granziera S, Cohen AT, Nante G, et al. Thromboembolic prevention in frail elderly patients with atrial fibrillation: a practical algorithm. J Am Med Dir Assoc 2015;16:358-64.

4. Fohtung RB, Novak E, Rich MV. Effect of new oral anticoagulants on prescribing practices for atrial fibrillation in older adults. J Am Geriatr Soc 2017;65:2405-12.

5. Bo M, Grisoglio E, Brunetti E, et al. Oral anticoagulant therapy for older patients with atrial fibrullation: a review of current evidence. Eur J Intern Med 2017;41:18-27.

6. Sardar P, Chatterjee S, Chaudhari S, Lip GY. New oral anticoagulants in elderly adults: evidence from a meta-analysis of randomized trials. J Am Geriatr Soc 2014;62:857-64

7. Sharma M, Cornelius VR, Patel JP, et al. Efficacy and harms of direct oral anticoagulants in the elderly for stroke prevention in atrial fibrillation and secondary prevention of venous thromboembolism: systematic review and meta-analysis. Circulation 2015;132:194-204.

8. Sadlon AH, Tsakiris DA. Direct oral anticoagulants in the elderly: systematic review and meta-analysis of evidence, current and future directions. Swiss Med Wkly 2016;146:w14356.

9. Bai Y, Guo SD, Deng $H$, et al. Effectiveness and safety of oral anticoagulants in older patients with atrial fibrillation: a systematic review and meta-regression analysis. Age Ageing 2018; 47:9-17.

10. Al-Khalili F, Lindström C, Benson L. Adherence to anticoagulant treatment with apixaban and rivaroxaban in a realworld setting. Clin Trials Regul Sci Cardiol 2016;18:1-4.

11. Jankowska-Polańska B, Katarzyna L, Lidia A, et al. Cognitive function and adherence to anticoagulation treatment in patients with atrial fibrillation. J Geriatr Cardiol 2016;13:559-65.

12. Mekaj YH, Mekaj AY, Duci SB, Miftari EI. New oral anticoagulants: their advantages and disadvantages compared with vitamin $\mathrm{K}$ antagonists in the prevention and treatment of patients 
with thromboembolic events. Ther Clin Risk Manage 2015;11:967-77.

13. Heidbuchel H, Verhamme P, Alings M, et al. EHRA Practical Guide on the use of new oral anticoagulants in patients with non-valvular atrial fibrillation: executive summary. Eur Heart J 2013; 34:2094-106.
14. Karamichalakis N, Georgopoulos S, Vlachos K, et al. Efficacy and safety of novel anticoagulants in the elderly. J Geriatr Cardiol 2016;13:718-23. 15. Orso F, Barucci R, Fracchia S, et al. Treatment of very old patients with non valvular atrial fibrillation The valuable opportunity offered by new oral anticoagulants, to be cautiously used. Monaldi Arch Chest Dis 2013; 80:151-60.

16. Alboni P, Stucci N, Cojocaru E, et al. Efficacia e sicurezza degli anticoagulanti orali nell'anziano fragile con fibrillazione atriale: problema ancora aperto. G Ital Cardiol 2017;18:180-87. 